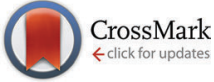

Cite this: Phys. Chem. Chem. Phys., $2015,17,26670$

Received 23rd July 2015, Accepted 7th September 2015

DOI: $10.1039 / c 5 c p 04312 c$

www.rsc.org/pccp

\title{
Mechanisms of $\mathrm{S}_{\mathrm{N}} 2$ reactions: insights from a nearside/farside analysis
}

\author{
Carsten Hennig and Stefan Schmatz* \\ A nearside/farside analysis of differential cross sections has been performed for the complex-forming \\ $\mathrm{S}_{\mathrm{N}} 2$ reaction $\mathrm{Cl}^{-}+\mathrm{CH}_{3} \mathrm{Br} \rightarrow \mathrm{ClCH}_{3}+\mathrm{Br}^{-}$. It is shown that for low rotational quantum numbers a direct \\ "nearside" reaction mechanism plays an important role and leads to anisotropic differential cross \\ sections. For high rotational quantum numbers, indirect mechanisms via a long-lived intermediate complex \\ are prevalent (independent of a nearside/farside configuration), leading to isotropic cross sections. Quantum \\ mechanical interference can be significant at specific energies or angles. Averaging over energies and angles \\ reveals that the nearside/farside decomposition in a semiclassical interpretation can reasonably account for \\ the analysis of the reaction mechanism.
}

\section{Introduction}

State-resolved differential cross sections for chemical reactions, containing information on angular distributions of the scattered products and thus describing a reactive process at a very detailed level, provide the most stringent test for a quantitatively accurate theoretical model for elementary chemical reactions. ${ }^{1-6}$ Only a few reactive systems could be studied with quantum-state resolution in scattering experiments, ${ }^{7-14}$ where the flux of the reaction products is measured that go into different scattering angles and final ro-vibrational states for different initial levels. Differential cross sections often exhibit complicated interference patterns ${ }^{1-5,15}$ which are difficult to analyse. Ion-molecule reactions, studied experimentally employing crossed-beam imaging, ${ }^{15}$ an approach widely used for reactions between neutral species, ${ }^{3,16}$ are also employed for the investigation of reactions between neutrals and anions, based on the technique of velocity map imaging. ${ }^{17} \mathrm{~A}$ velocity map imaging spectrometer in conjunction with a low-energy ion source has been used successfully to study gas-phase $\mathrm{S}_{\mathrm{N}} 2$ reactions by Wester and co-workers. ${ }^{18}$

Several reaction mechanisms are possible in the prototypical $\mathrm{S}_{\mathrm{N}} 2$ reaction $\mathrm{X}^{-}+\mathrm{CH}_{3} \mathrm{Y} \rightarrow \mathrm{XCH}_{3}+\mathrm{Y}^{-}$with halogen atoms $\mathrm{X}$ and $\mathrm{Y}^{19-25}$ In the direct mechanism, the nucleophile $\mathrm{X}^{-}$ directly attacks the cone built by the carbon and the three hydrogen atoms and forms the new $\mathrm{X}-\mathrm{C}$ bond in a direct collision while simultaneously the other bond, $\mathrm{C}-\mathrm{Y}$, is broken. In the indirect mechanism, on the other hand, a relatively longlived resonance state is produced, corresponding to an intermolecular complex that is bound by long-range ion-dipole forces.

Institut für Physikalische Chemie, Universität Göttingen, Tammannstr. 6, D-37077 Göttingen, Germany.E-mail: sschmat@gwdg.de
This complex carries out rotations before dissociating back into the reactants or finally forming the products. By investigating the importance of different reaction channels, new insight can be gained into this fundamental chemical reaction mechanism.

In our previous quantum-mechanical studies on nucleophilic bimolecular substitutions, ${ }^{26-32}$ reaction probabilities and total reaction cross sections have been calculated and discussed in detail. In ref. 32, differential cross sections have been computed based on rotationally resolved time-independent quantummechanical scattering calculations for $\mathrm{Cl}^{-}+\mathrm{CH}_{3} \mathrm{Br} \rightarrow \mathrm{ClCH}_{3}+$ $\mathrm{Br}^{-}$. The results show almost isotropic cross sections for reactant molecules with high rotational quantum numbers. Backward scattering is disfavoured for reaction out of states with small rotational excitation, in particular the rovibrational ground state. This is due to a quantum-mechanical effect, the interference of partial waves, that in general can be rationalized by simple classical arguments. In particular for higher vibrational excitation, an umbrella effect that favours the backward direction can be explained by the strong enhancement of the reactivity by opening a direct reaction mechanism. In non-central collisions, or in other words collisions with initial rotational motion, a torque due to the ion-dipole interaction is exerted on the molecule which after a rotation by about $90^{\circ}$ completes the reaction.

Connor and co-workers for the first time applied an approximate version of Fuller's nearside/farside theory of angular scattering originally developed to understand nuclear heavy-ion differential elastic cross sections - to molecular collisions. ${ }^{33}$ While the nearside/farside theory provides valuable insight into structured angular distributions, a disadvantage of a nearside/farside decomposition is that it is not unique.

In the present paper, we report on a nearside/farside analysis in the prototypical substitution reaction $\mathrm{Cl}^{-}+\mathrm{CH}_{3} \mathrm{Br} \rightarrow \mathrm{ClCH}_{3}+$ $\mathrm{Br}^{-}$. From a theoretical point of view, it is necessary to extract 
converged complex $S$-matrix elements from a quantum scattering calculation, not only the squared moduli in which the phase information is lost. While the latter are sufficient for the calculation of integral scattering cross sections (and can be obtained by averaging without taking into account the phases), differential cross sections are computed by first summing up the $S$-matrix elements weighted by Legendre polynomials and squaring the moduli of that sums afterwards. The complex $S$-matrix elements are obtained from the scattering wavefunction after projecting the asymptotic wavefunction onto channel Jacobi coordinates.

The paper is organized as follows: in Section II we report on the theory behind the nearside/farside analysis as applied to a molecular collision, while numerical details are contained in Section III. The results and their discussion are presented in Section IV. Finally, Section V gives our conclusions. Throughout, all energies are quoted in $\mathrm{cm}^{-1}$.

\section{Theory}

The computation of $S$-matrix elements for the reaction $\mathrm{Cl}^{-}+$ $\mathrm{CH}_{3} \mathrm{Br}(v, j) \rightarrow \mathrm{ClCH}_{3}\left(v^{\prime}, j^{\prime}\right)+\mathrm{Br}^{-}$is described in detail in ref. 31 and 32 . In ref. 32, state-resolved differential cross sections at total energy $E$ for vanishing helicity have been computed from $S$-matrices $S_{n m}^{J}(E)$ employing the usual formula

$$
\frac{\mathrm{d} \sigma_{n m}}{\mathrm{~d} \Omega}(E, \theta)=\frac{1}{4 k_{n}^{2}}\left|f_{n m}(E, \theta)\right|^{2},
$$

with the scattering amplitude $f(E, \theta)$ given by a partial wave summation

$$
f_{n m}(E, \theta)=\sum_{J=0}^{\infty} a_{n m, J}(E) P_{J}(\cos (\theta))
$$

and coefficients $a_{n m, J}(E)$ according to

$$
a_{n m, J}(E)=(2 J+1) S_{n m}^{J}(E) .
$$

Here, $k_{n}$ denotes the wavenumber of the initial state and $P_{J}$ is the usual Legendre polynomial of the first kind of order $J . \theta=0$ denotes forward scattering, and $\theta=\pi$ corresponds to backward scattering.

The differential cross sections themselves can be directly related to experimental results. To shed more light on the underlying reaction mechanisms, we employed a nearside/ farside analysis introduced by Fuller ${ }^{34}$ which has successfully been applied to reactive scattering. ${ }^{33,35}$ In a nearside/farside analysis, the scattering amplitude is decomposed into a sum of two subamplitudes moving clockwise $\left(f^{-}(\theta)\right)$ and counterclockwise $\left(f^{+}(\theta)\right)$ around the interaction zone:

$$
f(\theta)=f^{+}(\theta)+f^{-}(\theta)
$$

with $f^{ \pm}(\theta)$ defined by

$$
f^{ \pm}(\theta)=\sum_{J=0}^{\infty} a_{J} P_{J}^{ \pm}(\cos (\theta)) .
$$

In a semiclassical picture, $f^{-}(\theta)$ can be associated with trajectories scattered from the nearside of the target (with respect to

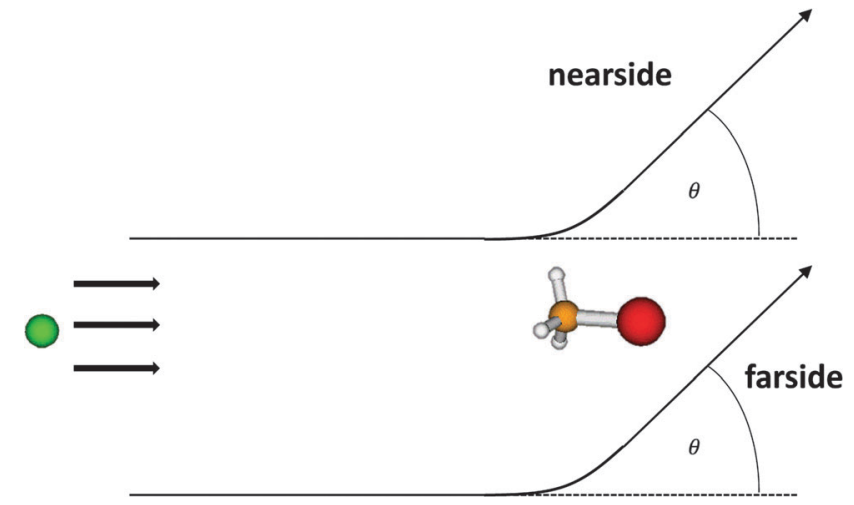

Fig. 1 Nearside and farside scattering for the $\mathrm{S}_{\mathrm{N}} 2$ reaction $\mathrm{Cl}^{-}+\mathrm{CH}_{3} \mathrm{Br} \rightarrow$ $\mathrm{ClCH}_{3}+\mathrm{Br}^{-}$. Two semiclassical trajectories with the same scattering angle, i.e. equally contributing to the differential cross section are shown. The nearside trajectory runs nearer to the detector which is thought to be positioned at the angle $\theta$. See the text for comments on this simplified picture since for collisions beyond the simple elastic atom-atom case the semiclassical nearside/farside theory is not trivial.

the detector), while $f^{+}(\theta)$ corresponds to trajectories on the farside of the target. The two semiclassical trajectories contribute to the same scattering angle. Applying this picture to the $\mathrm{Cl} / \mathrm{Br}$ exchange reaction, a direct reaction mechanism is expected to contribute mainly to the nearside scattering amplitude, while an indirect mechanism (with the formation of a long-lived complex) contributes to both the nearside and the farside amplitude. This is schematically shown in Fig. 1. Here, it should be noted that semiclassical trajectories can be easily displayed for simple non-reactive atom-atom collisions, where the change of the scattering vector is monitored. In this case, nearside and farside trajectories leading to the same scattering angle show different angles with respect of the rotation of the scattering vector ( $c f$. Fig. 1 in ref. 36). However, when more than two particles are involved, the task of identifying nearside and farside contributions is not trivial. For non-reactive atomdiatom collisions, a similar procedure can be applied employing the projection of the Jacobi vector onto the fixed plane perpendicular to the total angular momentum vector; its precession angle during the course of the collision is then monitored. ${ }^{36}$ This formalism can be extended relatively simply to reactive atom-diatom collisions. ${ }^{36}$ Thus, the trajectories shown in Fig. 1 are not easily comparable to curves directly related to geometrical data obtained from a conventional semiclassical calculation.

However, the decomposition of the scattering amplitude into subamplitudes is not unique. The final differential cross section is composed of contributions from nearside scattering, farside scattering and interferences of both. In the present analysis, we use the canonical decomposition

$$
P_{J}^{ \pm}(\cos (\theta))=\frac{1}{2}\left(P_{J}(\cos (\theta)) \mp \frac{2 i}{\pi} Q_{J}(\cos (\theta))\right)
$$

with $Q_{J}(\cos (\theta))$ being Legendre polynomials of the second kind. Substituting eqn (4) into eqn (1), we obtain a decomposition of 
the differential cross section into a farside (superscript + ), a nearside $(-)$ and an interference $(i)$ component:

$$
\frac{\mathrm{d} \sigma_{n m}}{\mathrm{~d} \Omega}(E, \theta)=\frac{\mathrm{d} \sigma_{n m}^{+}}{\mathrm{d} \Omega}(E, \theta)+\frac{\mathrm{d} \sigma_{n m}^{-}}{\mathrm{d} \Omega}(E, \theta)+\frac{\mathrm{d} \sigma_{n m}^{i}}{\mathrm{~d} \Omega}(E, \theta),
$$

where the nearside/farside components are given by

$$
\frac{\mathrm{d} \sigma_{n m}^{ \pm}}{\mathrm{d} \Omega}(E, \theta)=\frac{1}{4 k_{n}^{2}}\left|f_{n m}^{ \pm}(E, \theta)\right|^{2}
$$

while the interference component results from

$$
\frac{\mathrm{d} \sigma_{n m}^{i}}{\mathrm{~d} \Omega}(E, \theta)=\frac{1}{4{k_{n}}^{2}}\left(f_{n m}^{+}(E, \theta) \overline{f_{n m}^{-}(E, \theta)}+\overline{f_{n m}^{+}(E, \theta)} f_{n m}^{-}(E, \theta)\right) .
$$

with the bars denoting complex conjugation.

\section{Numerical details}

The $\mathrm{S}_{\mathrm{N}} 2$ reaction is treated as a pseudo-triatomic system with the methyl group regarded as a united atom. The internal geometry of this group is optimised for each set of Jacobi coordinates $R, r$ and $\gamma$. Starting with the exact Hamiltonian operator in Jacobi coordinates and restricting ourselves to vanishing helicity, we drop the Coriolis coupling term (centrifugal sudden approximation, $\mathrm{CS}^{37,38}$ ) and obtain the Hamiltonian for total angular momentum $J>0$

$$
\hat{H}_{\mathrm{CS}}=\hat{T}^{J=0}+\hat{T}_{\mathrm{CS}}^{J}+\hat{V},
$$

with the influence of total angular momentum approximated in the kinetic energy contribution $\hat{T}_{\text {CS }}^{J}$ according to

$$
\hat{T}_{\mathrm{CS}}^{J}=\delta_{j^{\prime} j} \frac{\hbar^{2}}{2 \mu_{1} R^{2}} J(J+1)
$$

where $j$ denotes the angular momentum of the pseudo-diatom.

In the framework of time-independent quantum scattering theory the Schrödinger equation for fixed total energy $E$ and fixed $J$ is solved employing an expansion of the wavefunction in products of translational functions and eigenfunctions of the surface Hamiltonian. The numerical solution of the scattering problem makes use of the technique of $R$-matrix propagation. ${ }^{39}$ We employ the wave functions and overlap matrices computed for $J=0$ and add the above term $\hat{T}_{\mathrm{CS}}^{J}$ as an energetic correction to the Hamiltonian in the propagation of the wavefunction along the hyperradius. Asymptotically we impose appropriate boundary conditions and finally obtain the complex scattering matrix.

We made use of the same parameters as employed in ref. 31 and 32 . To be able to compare the results, we carry out the calculations at the same energies for nearside/farside stateresolved differential cross sections as in ref. 32: $E_{\text {dif }}=350,850$, 1400 and $1900 \mathrm{~cm}^{-1}$; to smoothen many fluctuations that are characteristic of this resonance-driven reaction, for each energy $E_{\text {dif }}$, differential cross sections were actually computed at 21 equidistant values $E_{\text {dif }}-1.0 \mathrm{~cm}^{-1}, E_{\text {dif }}-0.9 \mathrm{~cm}^{-1}, \ldots, E_{\text {dif }}+$ $1.0 \mathrm{~cm}^{-1}$ and then averaged. In addition, for each angle $\theta_{\text {dif }}$ the differential cross sections have been averaged over intervals of $\left[\theta_{\mathrm{dif}}-\theta_{\mathrm{av}}, \theta_{\mathrm{dif}}+\theta_{\mathrm{av}}\right]$, with $\theta_{\mathrm{av}}=5^{\circ}$.

\section{Results and discussion}

In Fig. 2, 3, 5 and 6, the initial-state selected differential reaction cross sections and the corresponding nearside/farside decompositions are graphically displayed. They are summed over all product states for total energies $E_{\text {tot }}$ of $350,850,1400$ and $1900 \mathrm{~cm}^{-1} \cdot \sin (\theta)(\mathrm{d} \sigma / \mathrm{d} \Omega)\left(E_{\text {tot }}\right)$ is shown, which is the integrand employed in the calculation of the total cross sections $\sigma_{\text {tot }}\left(E_{\text {tot }}\right)$, making the contributions to $\sigma_{\text {tot }}$ visible. As a consequence, the data for $\theta=0$ and $\theta=180^{\circ}$ are exactly zero, as long as the differential cross sections remain finite. This is not seen in the plots, first because this occurs only at precisely these two angles and second because of data averaging. The data are smoothly averaged over intervals of $10^{\circ}$ with the exception of 0 and $180^{\circ}$. Forward scattering with the newly formed $\mathrm{Br}^{-}$ion moving to the left corresponds to $\theta=0$, while backward scattering with that ion moving to the right is associated with $\theta=180^{\circ}$.

Fig. 2 shows the differential reaction cross sections for a total energy of $350 \mathrm{~cm}^{-1}$ and different initial rotational excitations
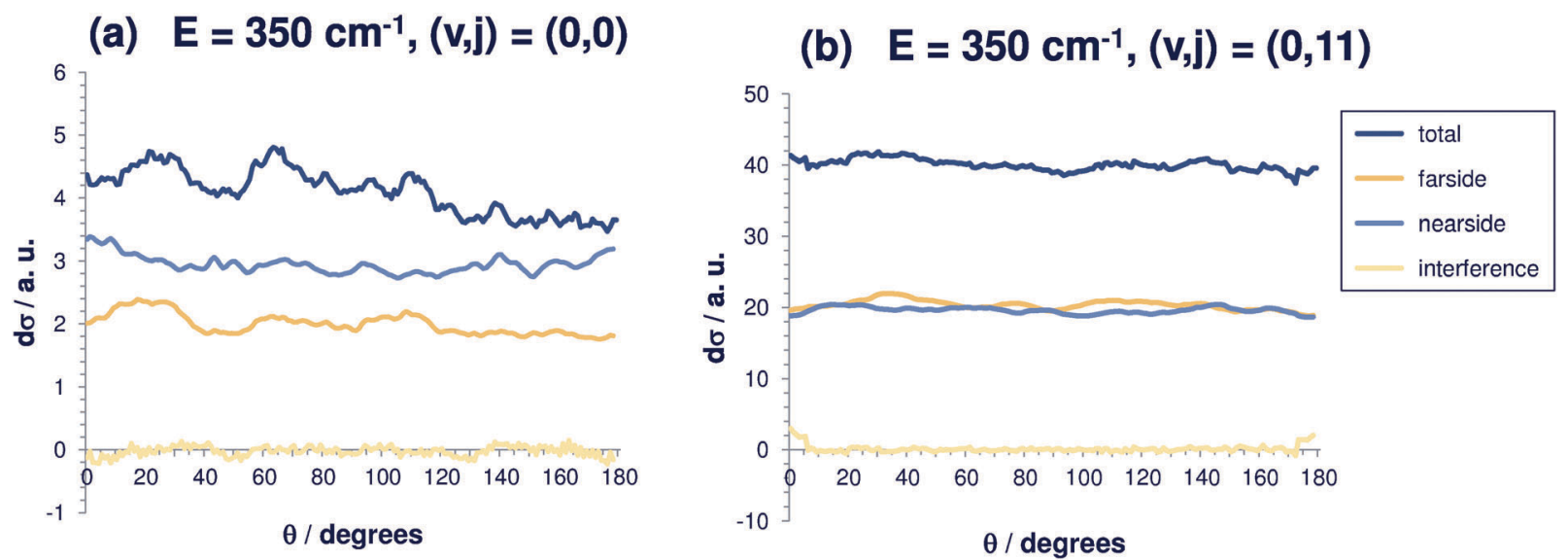

Fig. 2 Differential reaction cross sections for $\mathrm{Cl}^{-}+\mathrm{CH}_{3} \mathrm{Br}(v, j) \rightarrow \mathrm{ClCH}_{3}+\mathrm{Br}^{-}$at $E=350 \mathrm{~cm}^{-1}$ and decomposition in nearside and farside contributions for selected initial rovibrational states $(v, j)$. Also shown is the interference contribution. $(a)(v, j)=(0,0) ;(b)(v, j)=(0,11)$. 

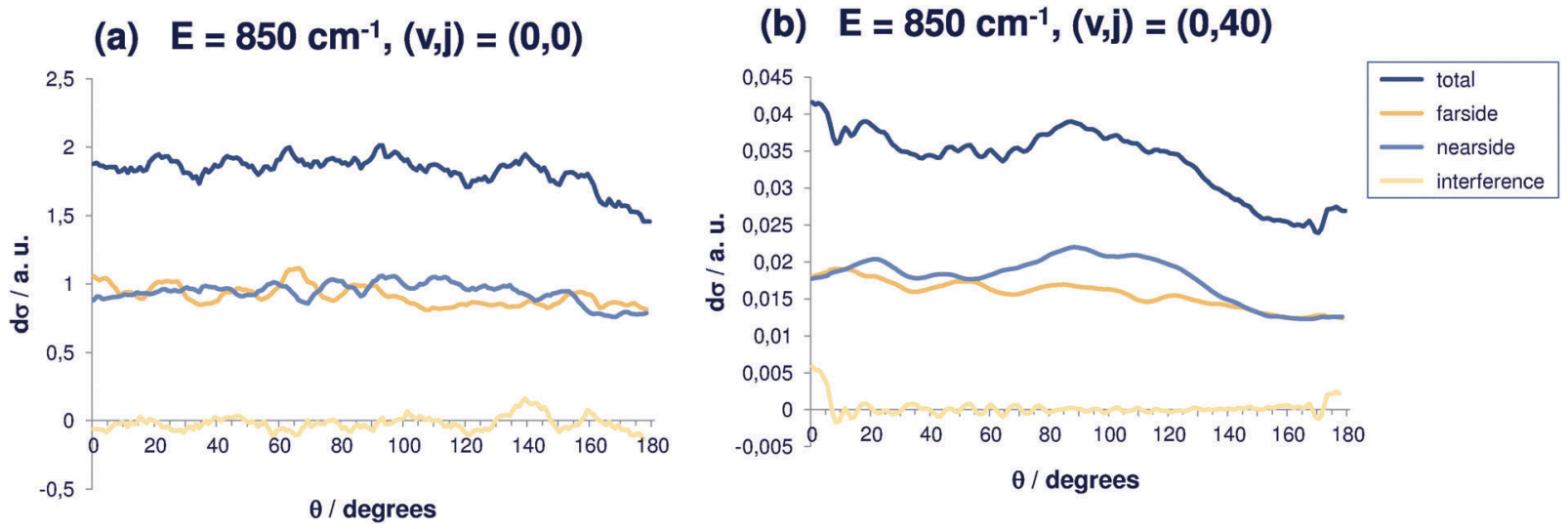

Fig. 3 Differential reaction cross sections for $\mathrm{Cl}^{-}+\mathrm{CH}_{3} \mathrm{Br}(v, j) \rightarrow \mathrm{ClCH}_{3}+\mathrm{Br}^{-}$at $E=850 \mathrm{~cm}^{-1}$ and decomposition in nearside and farside contributions for selected initial rovibrational states $(v, j)$. Also shown is the interference contribution. (a) $(v, j)=(0,0) ;(b)(v, j)=(0,40)$.

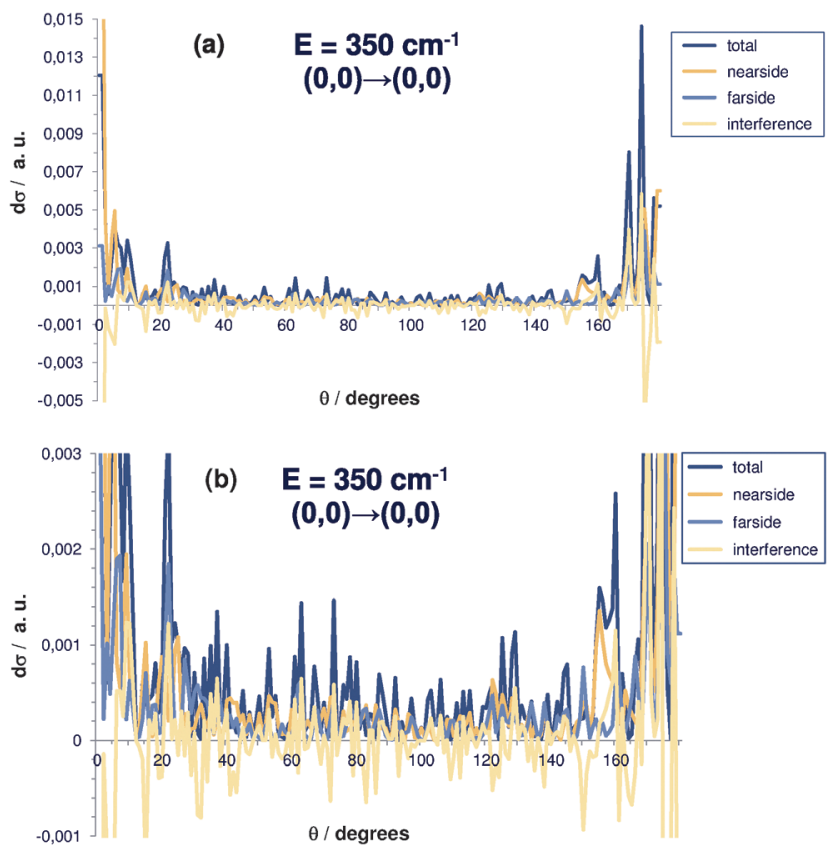

Fig. 4 (a) State-to-state specific differential reaction cross section for $\mathrm{Cl}^{-}+\mathrm{CH}_{3} \mathrm{Br}(0,0) \rightarrow \mathrm{ClCH}_{3}(0,0)+\mathrm{Br}^{-}$at $E=350 \mathrm{~cm}^{-1}$ and decomposition in nearside, farside and interference contributions. (b) Same as (a), but detail with enlarged scale along the vertical coordinate axis. Note the negative and positive parts in the interference contribution

$(v=0 ; j=0,11)$. At this relatively small energy, it can be seen clearly that $(\mathrm{d} \sigma / \mathrm{d} \Omega) \sin (\theta)$ increases with $j$ (note the scaling) and becomes increasingly more isotropic. For small $j$, many oscillations typical of resonance features can be observed. Here, the fact that the cross sections become larger with $j$ can be explained mainly by an energetic effect. Rotationally excited initial states are higher in energy, and thus the systems have at a given total energy - less translational energy available which, however, enters the equation for the calculation of the cross section in the denominator. For the initial state $(0,0)$, back scattering is slightly less effective. For low total energies, the nearside/farside analysis reveals some structure in the differential cross sections with a slight preference of small scattering angles. For initially vibrationless and rotationless states the nearside scattering dominates the farside contribution by $20 \%$, partly by more than $50 \%$, pointing towards a preference of the direct reaction mechanism over complexformation.

However, if rotation is excited, the relative difference between nearside and farside contribution becomes negligible. The interference contribution between the nearside and the farside amplitude is very small. For higher rotational excitation, this effect becomes even more pronounced, indicating that if the methyl bromide molecule rotates, the reaction occurs mainly via complex-formation and not via the direct reaction pathway. So, the differential cross section becomes isotropic for high initial rotational excitation.

In agreement with our previous results,${ }^{30}$ the differential cross sections are largest for small energies. At higher total energies $\left(850 \mathrm{~cm}^{-1}\right.$, Fig. 3$)$, the $(0,0)$ differential cross section shows a similar behavior, i.e. back scattering being slightly less probable, but the average differential cross section has become smaller by roughly a factor of two. Also, the oscillations in the differential cross sections are less pronounced. For initial rotational excitation, the differential cross sections decrease and become more isotropic, so that finally almost no anisotropy can be observed (the cross section for angular momentum quantum number $j=40$ is shown in Fig. 3(b)). Isotropic cross sections point to the existence of long-living resonance states which shows that the correlation with the initial angle disappears. For reaction out of the ground state and out of the state with rotational excitation $j=40$, the reaction cross sections are anisotropic, the coarse-grained fluctuation is more pronounced for $j=40$ while the fine structure is less distinct. The scale of the cross sections in Fig. 3 should be noted. Again, nearside and farside scattering contributions are equally strong and overall the interference contribution obviously is negligible.

However, it should be kept in mind that the curves shown are averaged due to strong oscillating patterns in the cross sections for this resonance-driven reaction. Also in the integral cross sections calculated within a model with the number of 

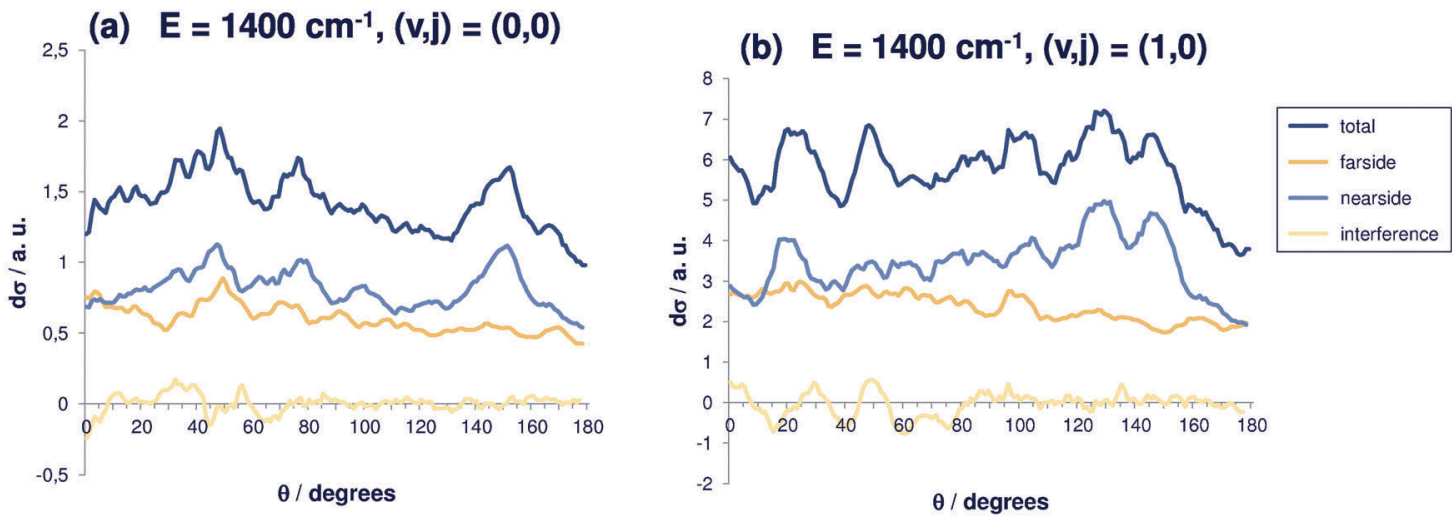

(c) $E=1400 \mathrm{~cm}^{-1},(\mathrm{v}, \mathrm{j})=(0,42)$

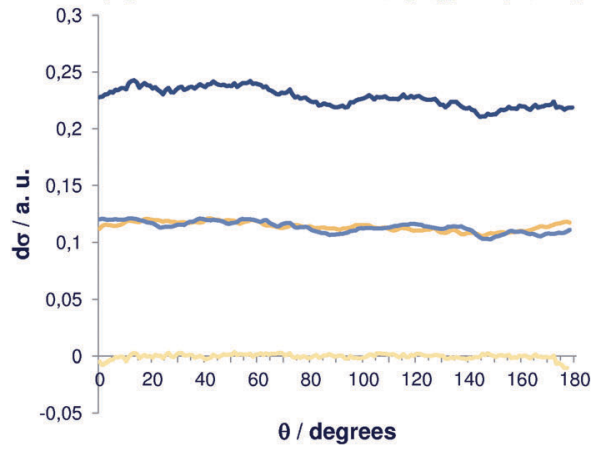

(d) $E=1400 \mathrm{~cm}^{-1},(v, j)=(1,38)$

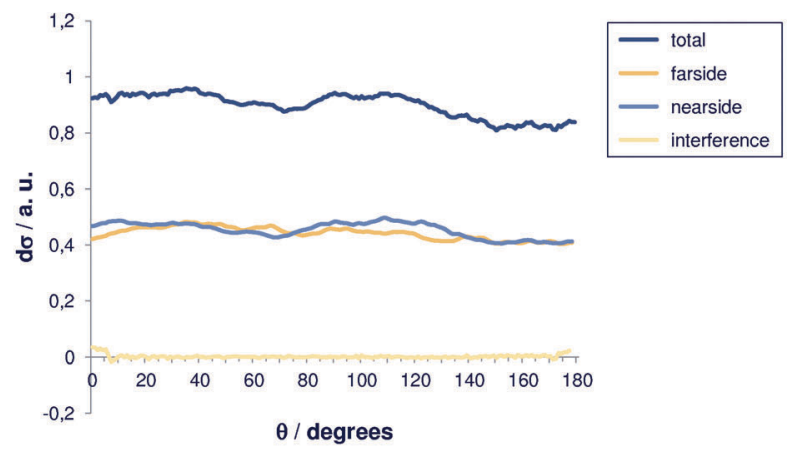

Fig. 5 Differential reaction cross sections for $\mathrm{Cl}^{-}+\mathrm{CH}_{3} \mathrm{Br}(v, j) \rightarrow \mathrm{ClCH}_{3}+\mathrm{Br}^{-}$at $E=1400 \mathrm{~cm}^{-1}$ and decomposition in nearside and farside contributions for selected initial rovibrational states $(v, j)$. Also shown is the interference contribution. (a) $(v, j)=(0,0) ;(b)(v, j)=(1,0) ;(c)(v, j)=(0,42) ;(d)(v, j)=(1,38)$.

degrees of freedom reduced to only two, many extremely narrow resonances are present; an even more pronounced resonance structure is found in the differential cross section. Furthermore, the differential cross sections shown in the present paper are summed over product states, and this summation process smoothens the curves presented in this paper.

The raw data, i.e. state-to-state cross sections before averaging over energies and angles, show prominent interference patterns. This can be seen from Fig. 4 for the state-to-state differential cross section at a fixed energy of $350 \mathrm{~cm}^{-1}$ as a function of the scattering angle (the $(0,0) \rightarrow(0,0)$ transition is shown). In particular, while the total, nearside and farside contributions are strictly positive due to their definition, the interference term is real, but can be negative as can be clearly seen from Fig. 4. After averaging, the interference contribution becomes negligible. This to a large extent is due to the annihilation of negative and positive components. That justifies the interpretation of this term as an interference type component of the differential cross section and helps to identify the reaction mechanisms in a semiclassical picture and to establish the link to experimental results.

For even higher total energy (Fig. 5(a)), $1400 \mathrm{~cm}^{-1}$, similar features can be observed so that the trend becomes even clearer. However, the coarse oscillations for $(0,0)$ are again more pronounced. Fig. 5(b) shows the same for additional initial vibrational excitation, which is possible at this given total energy. The differential cross sections increase as expected from our previous collinear studies, ${ }^{30}$ while the behaviour with respect to angular excitation remains as before. The dip for backward scattering becomes more pronounced and the oscillations are coarser. For reaction out of the ground state and the state with one quantum in the $\mathrm{C}-\mathrm{Br}$ stretching vibration, the differential cross sections show structure, but not a clear preference of a particular angular regime. Nearside scattering dominates, in particular for $(v, j)=(1,0)$ and for wide angles, whereas for small angles the contributions of nearside and farside scattering are very similar. A relatively strong interference contribution is observed. Clearly, the direct mechanism is enhanced when the broken bond is excited $(v=1$, Fig. 5(a)). High rotational energy means low translational energy. The cross sections are almost perfectly isotropic and the nearside and farside contributions are very similar.

Again, for energies of $1900 \mathrm{~cm}^{-1}$ (Fig. 6(a-f)), the differential cross sections tend to be more isotropic for larger values of $j$. The cross section for the initial state $(0,0)$ becomes more and more interesting (Fig. 6(a)), because the peak for backward scattering $\left(\theta=180^{\circ}\right)$ is only one third as high as the maximum value located in the range between $100^{\circ}$ and $160^{\circ}$. The structural features become even coarser and the typical resonance structure is lost. Without initial excitation (Fig. 6(a)), the nearside part is strongly dominating. The scattering occurs in the angular range between $100^{\circ}$ and $170^{\circ}$. The farside contribution remains almost constant, which points towards a direct reaction mechanism. Additional vibrational excitation (Fig. 6(b)) 
(a) $E=1900 \mathrm{~cm}^{-1},(v, j)=(0,0)$

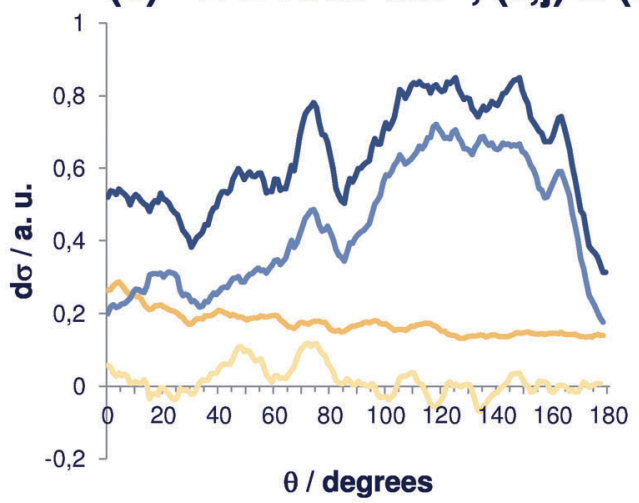

(c) $E=1900 \mathrm{~cm}^{-1},(\mathrm{v}, \mathrm{j})=(2,0)$

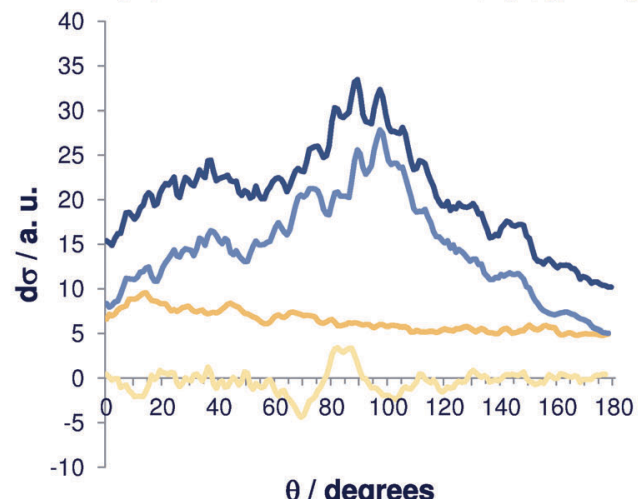

(e) $E=1900 \mathrm{~cm}^{-1},(\mathrm{v}, \mathrm{j})=(2,34)$

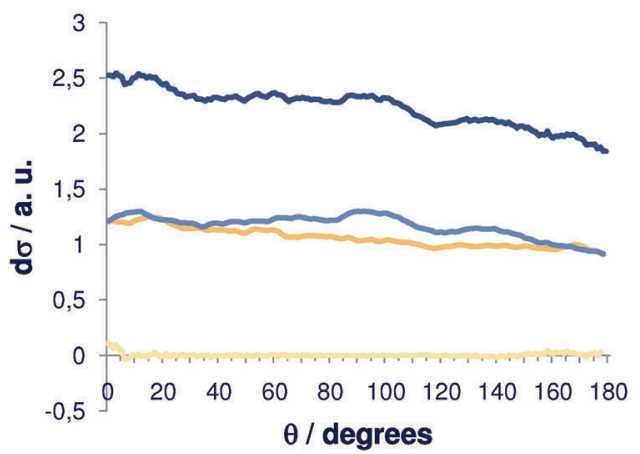

(b) $\quad E=1900 \mathrm{~cm}^{-1},(v, j)=(1,0)$

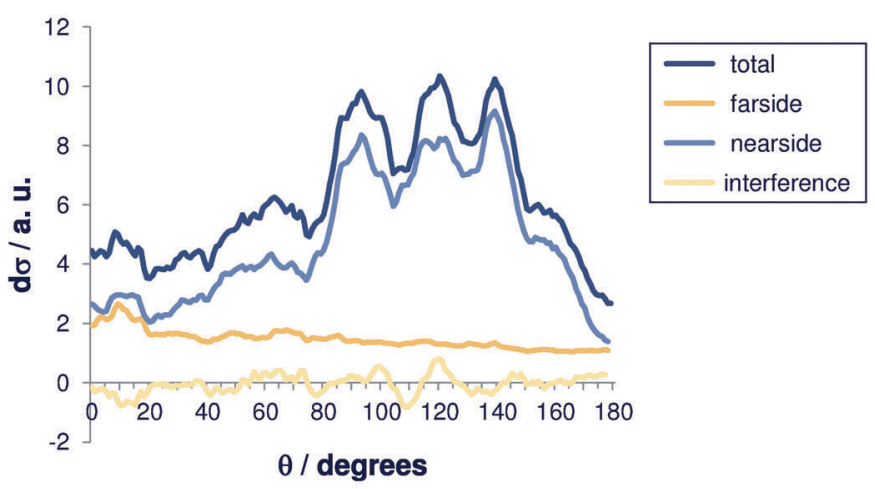

(d) $\quad E=1900 \mathrm{~cm}^{-1},(v, j)=(1,42)$

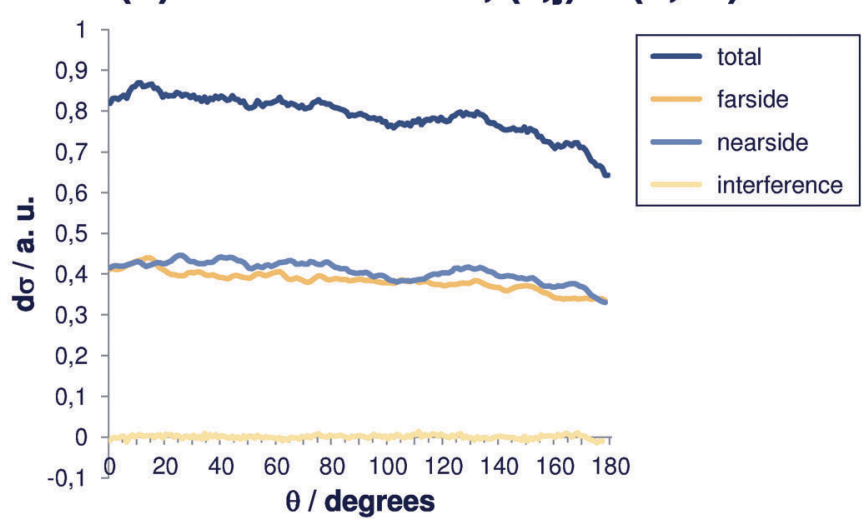

(f) $E=1900 \mathrm{~cm}^{-1},(v, j)=(0,69)$

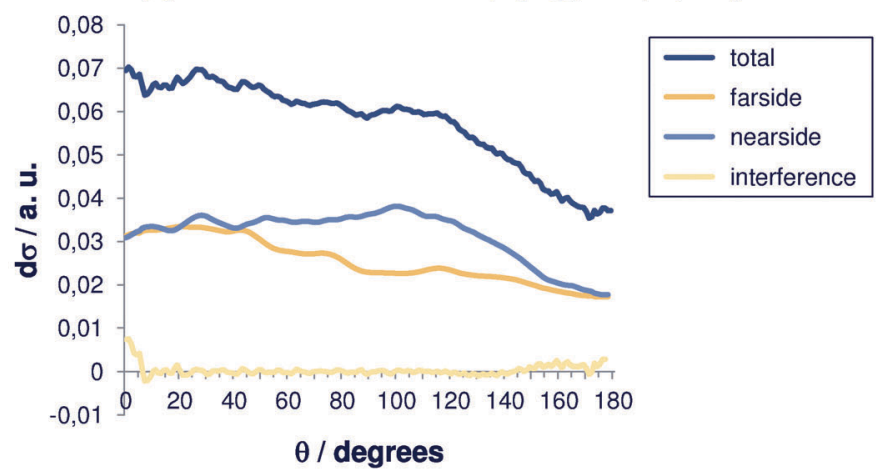

Fig. 6 Differential reaction cross sections for $\mathrm{Cl}^{-}+\mathrm{CH}_{3} \mathrm{Br}(v, j) \rightarrow \mathrm{ClCH}_{3}+\mathrm{Br}^{-}$at $E=1900 \mathrm{~cm}^{-1}$ and decomposition in nearside and farside contributions for selected initial rovibrational states $(v, j)$. Also shown is the interference contribution. (a) $(v, j)=(0,0) ;(b)(v, j)=(1,0) ;(c)(v, j)=(2,0) ;(d)(v, j)=(1,42) ;(e)(v, j)=$ $(2,34)$; (f) $(v, j)=(0,69)$.

shifts this range to $90^{\circ}-140^{\circ}$ with backward scattering less than one third of the maximum cross section. An even larger effect is seen in Fig. 6(b) with a pronounced interference pattern. Two quanta in the vibrational mode cause a further shift down to $80^{\circ}-115^{\circ}$. For two quanta in the $\mathrm{C}-\mathrm{Br}$ stretching vibration, $(v, j)=$ $(2,0)$ (Fig. 6(c)), an angular maximum at $90^{\circ}$ is observed. For even higher initial energies $\left(1900 \mathrm{~cm}^{-1}\right)$ the differential cross sections are shown in Fig. 6(d). For high rotational excitation $(v=42)$, the same effect as already discussed occurs. The same can be said for Fig. 6(e). From Fig. 6(f), we see that the forward scattering is reduced and the nearside contribution again dominates.

To rationalize the calculated behaviour of the differential reaction cross sections, in particular the isotropy for large $j$-values, it is useful to go back to simple classical-mechanical arguments. For a fastly rotating target molecule, all directions for dissociation of the intermediate complex are possible and equally probable. A complex that lives long enough (i.e. the resonance of which is narrow enough with respect to energy) 
can perform several rotations before the actual reaction takes place. So, no direction is favoured, yielding isotropic differential cross sections.

It should be discussed why cross sections for backward scattering are smaller. Remember that in our previous collinear studies $^{28-30}$ only backward scattering was possible, with the chloride anion attacking the methyl group, forming a bond with the carbon atom, and after the umbrella flip of the methyl group bouncing back to the direction where the chloride ion came from. In the full three-dimensional picture, this direction is strongly disfavoured because the chlorine anion approaches the $\mathrm{CH}_{3} \mathrm{Br}$ molecule off from the collinear axis. In the classicalmechanical picture, due to the strong ion-dipole interaction a torque is exerted on the molecule, and it begins to carry out a rotation or, when the ion is very close to the molecule, a libration. However, due to the shape of the potential, no full rotational cycle can be performed if the available energy is not high enough, and an angle of about $90^{\circ}$ seems most probable. This explains why exact backward scattering is such a disfavoured event. The strong electrostatic forces have a substantial influence on the differential cross sections in the cases without initial rotational excitation. The molecule orients towards the ion in the case of any deviation from the central collision.

\section{Conclusions}

We have carried out a nearside/farside analysis on the nucleophilic bimolecular substitution reaction $\mathrm{Cl}^{-}+\mathrm{CH}_{3} \mathrm{Br}(v, j) \rightarrow$ $\mathrm{ClCH}_{3}\left(v^{\prime}, j^{\prime}\right)+\mathrm{Br}^{-}$as it was first introduced in molecular scattering by Connor and co-workers based on an idea of Fuller. It is revealed that in gas-phase $\mathrm{S}_{\mathrm{N}} 2$ reactions the structure in the differential cross sections at higher energies is related to the nearside scattering contribution, pointing towards a direct reaction mechanism where the attacking ion directly approaches the open cone formed by the carbon and the three connected hydrogen atoms. The indirect mechanism, on the other hand, is described by complex-formation and subsequent dissociation after carrying out rotational motion. The differential cross section becomes isotropic because no angle is favoured. This mechanism clearly is connected with the farside contribution which introduces no structure in the differential reaction cross section.

\section{Acknowledgements}

This work was financially supported by the Deutsche Forschungsgemeinschaft (DFG), grant SCHM 1651/1-3. Additional support from the Fonds der chemischen Industrie is gratefully acknowledged.

\section{References}

1 D. C. Clary, Proc. Natl. Acad. Sci. U. S. A., 2008, 105, 12649.

2 D. C. Clary, Science, 2008, 321, 789.

3 K. Liu, Annu. Rev. Phys. Chem., 2001, 52, 139.

4 X. Yang, Annu. Rev. Phys. Chem., 2007, 58, 433.

5 W. Hu and G. C. Schatz, J. Chem. Phys., 2006, 125, 132301.
6 J. Mikosch, M. Wei and G. Nyman,J. Phys. Chem. A, 2010, 114, 9617. 7 P. D. D. Monks, J. N. L. Connor and S. C. Althorpe, J. Phys. Chem. A, 2006, 110, 741.

8 D. Dai, C. C. Wang, S. A. Harich, X. Wang, X. Yang, S. D. Chao and R. T. Skodje, Science, 2003, 300, 1730.

9 F. J. Aoiz, L. Banares, B. Martinez-Haya, J. Castillo, D. E. Manolopoulos, K. Stark and H.-J. Werner, J. Phys. Chem. A, 1997, 101, 6403.

10 M. Qiu, Z. Ren, L. Che, D. Dai, S. A. Harich, X. Wang, X. Yang, C. Xu, D. Xie, M. Gustafsson, R. T. Skodje, Z. Sun and D. H. Zhang, Science, 2006, 311, 1440.

11 J. N. L. Connor, Phys. Chem. Chem. Phys., 2004, 6, 377.

12 N. Balucani, D. Skouteris, L. Cartechini, G. Cappozza, E. Segeloni, P. Casavecchia, M. H. Alexander, G. Capecchi and H.-J. Werner, Phys. Rev. Lett., 2003, 91, 13201.

13 D. H. Zhang and J. Z. H. Zhang, J. Chem. Phys., 1994, 101, 1146. 14 E. J. Rackham, T. Gonzales-Lezana and D. E. Manolopoulos, J. Chem. Phys., 2003, 119, 12895.

15 J. Mikosch, M. Weidemüller and R. Wester, Int. Rev. Phys. Chem., 2010, 29, 589.

16 P. Casavecchia, Rep. Prog. Phys., 2000, 63, 355.

17 A. T. J. B. Eppink and D. H. Parker, Rev. Sci. Instrum., 1997, 68, 3477.

18 J. Mikosch, U. Frühling, S. Trippel, D. Schwalm, M. Weidemüller and R. Wester, Phys. Chem. Chem. Phys., 2006, 8, 2990.

19 W. L. Hase, Science, 1994, 266, 998.

20 M. L. Chabinyc, S. L. Craig, C. K. Regan and J. I. Brauman, Science, 1998, 279, 1882.

21 J. K. Laerdahl and E. Uggerud, Int. J. Mass Spectrom. Ion Processes, 2002, 214, 277.

22 S. Schmatz, ChemPhysChem, 2004, 5, 600.

23 J.-L. Le Garrec, B. R. Rowe, J. L. Queffelec, J. B. A. Mitchell and D. C. Clary, J. Phys. Chem., 1997, 107, 1021.

24 J. Mikosch, S. Trippel, C. Eichhorn, R. Otto, U. Lourderaj, J. X. Zhang, W. L. Hase, M. Weidemüller and R. Wester, Science, 2008, 319, 183.

25 J. Zhang and W. L. Hase, J. Phys. Chem. A, 2010, 114, 9635. 26 S. Schmatz and D. C. Clary, J. Chem. Phys., 1998, 109, 8200. 27 S. Schmatz and D. C. Clary, J. Chem. Phys., 1999, 110, 9483. 28 C. Hennig and S. Schmatz, J. Chem. Phys., 2004, 121, 220.

29 C. Hennig and S. Schmatz, J. Chem. Phys., 2005, 122, 234307. 30 C. Hennig and S. Schmatz, Chem. Phys. Lett., 2007, 446, 250. 31 C. Hennig and S. Schmatz, J. Chem. Phys., 2009, 121, 224303.

32 C. Hennig and S. Schmatz, Phys. Chem. Chem. Phys., 2012, 14(37), 12982.

33 J. N. L. Connor, P. McCabe, D. Sokolovski and G. C. Schatz, Chem. Phys. Lett., 1993, 206, 119.

34 R. C. Fuller, Phys. Rev. C: Nucl. Phys., 1975, 12, 1561.

35 A. J. Dobbyn, P. McCabe, J. N. L. Connor and J. F. Castillo, Phys. Chem. Chem. Phys., 1999, 1, 1115.

36 D. Sokolovski and J. N. L. Connor, Chem. Phys. Lett., 1999, 305, 238.

37 R. T. Pack, J. Chem. Phys., 1974, 60, 633.

38 P. McGuire and D. J. Kouri, J. Chem. Phys., 1974, 60, 2488.

39 E. B. Stechel, R. B. Walker and J. C. Light, J. Chem. Phys., 1978, 69, 3518. 